\title{
Patienten mit Tic-Störungen: weit bekannt, doch unterversorgt
}

Jaana M. L. Schnell ${ }^{*}$, Elif Weidinger*, Richard Musil

* geteilte Autorenschaft („,contributed equally“)

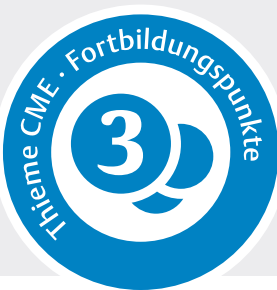

Das Bewusstsein der Öffentlichkeit für Tic-Störungen und insbesondere für das Tourette-Syndrom ist durch zahlreiche Medienbeiträge in den letzten Jahren gestiegen. Immer wieder wird in TV- oder Printmedien jedoch lediglich von schweren Fällen des Tourette-Syndroms berichtet, die nicht dem durchschnittlichen Krankheitsverlauf entsprechen. Eine umfangreiche Aufklärung über Symptome, Verlauf, Ursachen und Behandlungsmöglichkeiten ist daher wünschenswert [1].

\section{Definition}

Tics sind unwillkürliche, rasche, wiederholte, nicht-rhythmische Bewegungen meist umschriebener Muskelgruppen oder eine Lautproduktion, die plötzlich einsetzt und keinem erkennbaren Zweck dient.

Unterschieden werden

- vorübergehende Tic-Störungen (Dauer <12 Monate) (ICD-10: F95.0) und

- chronische Tic-Störungen (Dauer $\geq$ Nachdem hier ein "größergleich“ Zeichen schon steht, kann das „=“ Zeichen entfallen. 12 Monate) (F95.1 - F95.2).

Zu chronischen Tic-Störungen werden die chronische vokale Tic-Störung (ICD-10: F95.1), die chronische motorische Tic-Störung (ICD-10: F95.1) und das Tourette-Syndrom (Auftreten von motorischen und vokalen Tics, jedoch nicht zwingend gleichzeitig) (ICD-10: F95.2) gezählt [2].

Cave

Koprolalie (Aussprechen obszöner Inhalte) ist nicht Voraussetzung für die Diagnose eines TouretteSyndroms!

\section{INFOBOX 1}

Einteilung der Tic-Störungen nach ICD-10 (WHO 2011)

F 95.0 Vorübergehende Ticstörung

F 95.1 Chronische motorische oder vokale Ticstörung F 95.2 Kombinierte vokale und multiple motorische Tics (Tourette-Syndrom)

F 95.8 Sonstige Ticstörungen

F 95.9 Ticstörung, nicht näher bezeichnet

\begin{tabular}{|c|c|}
\hline \multicolumn{2}{|c|}{ ABKÜRZUNGEN } \\
\hline ADHS & $\begin{array}{l}\text { Aufmerksamkeitsdefizit-Hyperaktivi- } \\
\text { täts-Störung }\end{array}$ \\
\hline CBD & Cannabidiol \\
\hline $\mathrm{CB} 1 / \mathrm{CB} 2$ & Cannabinoid-Rezeptor $1 / 2$ \\
\hline CBIT & $\begin{array}{l}\text { Comprehensive Behavioral Interven- } \\
\text { tion for Tics }\end{array}$ \\
\hline CNV & Copy Number Variant \\
\hline DD2 / D3 & Dopamin-Rezeptor Typ 2 / 3 \\
\hline EEG & Elektroencephalographie \\
\hline ERP & Exposure and Response Prevention \\
\hline FGA & First Generation Antipsychotic \\
\hline HRT & Habit Reversal Training \\
\hline $5 \mathrm{HT} 1 \mathrm{~A}$ & Serotonin-Rezeptor Typ 1A \\
\hline $5 \mathrm{HT} 2 \mathrm{~A}$ & Serotonin-Rezeptor Typ 2A \\
\hline CMRT & cortikale \\
\hline & Magnetresonanztomographie \\
\hline PANDAS & $\begin{array}{l}\text { Pediatric Autoimmune Neuropsy- } \\
\text { chiatric Disorders Associated with } \\
\text { Streptococcal infections }\end{array}$ \\
\hline PANS & $\begin{array}{l}\text { Pediatric Acute Neuropsychiatric } \\
\text { Syndromes }\end{array}$ \\
\hline PUTS & Premonitory Urge for Tics Scale \\
\hline SGA & Second Generation Antipsychotic \\
\hline SNP & Single Nucleotide Polymorphism \\
\hline SSRI & $\begin{array}{l}\text { Selektive Serotonin Rückaufnahme- } \\
\text { Inhibitoren }\end{array}$ \\
\hline TGD & Tourette-Gesellschaft Deutschland \\
\hline THC & Tetrahydrocannabinol \\
\hline THS & Tiefenhirnstimulation \\
\hline VMAT & Vesikulärer Monoamintransporter \\
\hline YGTSS & Yale Global Tic Severity Scale \\
\hline
\end{tabular}




\section{Epidemiologie}

Tic-Störungen treten weltweit in gleicher Häufigkeit und gleicher Symptomatik auf [3]. Das Tourette-Syndrom hat eine Prävalenzrate von 0,85-1\%. Andere chronische und vorübergehende Tic-Störungen sowie einzelne Tics treten deutlich häufiger auf. So haben 10-15\% aller Grundschüler zu irgendeinem Zeitpunkt Tics. Jungen und Männer sind mit einem Verhältnis von 2:1 bis 4:1 deutlich häufiger betroffen als Mädchen und Frauen [4, 5].

\section{Ätiologie}

Die Ursache von Tic-Störungen konnte bislang nicht vollständig aufgeklärt werden. Die meisten Untersuchungen gelangen jedoch immer wieder zu einer grundlegenden Hypothese: einer Störung der kortiko-striato-pallido-thalamo-kortikalen Regelkreise. Einfacher ausgedrückt bedeutet dies, dass die zentrale Steuerung von Bewegungsabläufen in Mitleidenschaft gezogen ist.

Die Veränderungen in den kortiko-striato-pallido-thalamo-kortikalen Regelkreisen konnten zum einen durch nuklearmedizinische Methoden und zum anderen durch strukturelle und funktionelle MRT belegt werden.

So weisen z.B. PET-Untersuchungen auf Veränderungen in der Dichte an kortikalen und striatalen Dopaminrezeptoren sowie einer erhöhten Anzahl von Dopamintransportern hin [6]. Daneben zeigen insbesondere Antipsychotika [„Dopamin-Antagonisten“] eine positive therapeutische Wirkung auf Tics (siehe dazu auch den Abschnitt zur Pharmakotherapie). Beides begründet die derzeit vorherrschende „Dopamin-Hypothese“ von Tic-Störungen.

Auch einige andere Neurotransmittersysteme (v. a. Glutamat, GABA, Serotonin, Endocannabinoide, Acetylcholin) weisen Veränderungen auf, die jedoch bislang weniger stark beforscht wurden.

Strukturelle und funktionelle MRT-Untersuchungen zeigten eine Zunahme der Konnektivität z. B. des Putamen mit kortikalen und thalamischen Arealen sowie dem Globus pallidus. Ebenso weisen mehrere kortikale Regionen erhöhte Verbindungen mit dem Striatum und dem Thalamus auf. Eine erhöhte strukturelle Konnektivität motorischer Areale mit dem supplementärmotorischen Kortex korreliert zudem mit der TicSchwere-unabhängig von einer Medikamenteneinnahme, dem Alter oder Geschlecht [7].

Daneben zeigen sich die axonalen Faserverbindungen der weißen Substanz (z. B. Corpus callosum oder vordere Kapsel) verändert.

Zusammengefasst könnten diese Veränderungen Ausdruck einer Hirnentwicklungsstörung als mögliche Ursache des Tourette-Syndroms sein.
Verstärkte Aufmerksamkeit erhalten in den letzten Jahren die neuroanatomischen Korrelate der sogenannten Vorgefühle („premonitory urges“ [4]), die Tics häufig vorangehen. Die Entstehung dieser Vorgefühle wird auf eine Veränderung des Zusammenspiels sensorischer Kortexabschnitte mit der Inselregion und supplementär motorischen Kortexabschnitten zurückgeführt [8]. Die Erforschung der Entstehung der Vorgefühle ist von herausragender Bedeutung: Tics können als entlastende Reaktion auf diese unangenehmen Vorgefühle aufgefasst werden. Somit wären die Vorgefühle die eigentliche Ursache und die Tics bereits ein erlerntes Verhalten, damit umzugehen. Diese Zusammenhänge macht man sich bei einigen Psychotherapieansätzen (wie dem Habit Reversal Training (HRT) oder dem Exposure and Response Prevention Training (ERP)) von Tics zunutze [4].

Große Unklarheit besteht noch darüber, was zu den gestörten Regelkreisabläufen führt [9]. Im Wesentlichen werden genetische als auch Umwelteinflüsse diskutiert.

\section{Genetik}

Sicherlich tragen genetische Veränderungen einen erheblichen Teil zur Entstehung von Tics bei, wie aus Zwillings- und Adoptionsstudien hervorgeht. Grundsätzlich kann man von einer polygenen Ätiologie ausgehen: Eine Vielzahl von veränderten Genabschnitten leistet jeweils einen kleinen Beitrag zur Symptomentwicklung. So ist es auch nicht verwunderlich, dass in den durchgeführten genomweiten Assoziationsstudien bislang kein Genort eine genomweite Signifikanz $\left(p<5 \times 10^{-8}\right)$ erreichte. Der beste Kandidat lag im Intron für das Gen der Kollagen- $\alpha 1$-Kette (COL27A1; SNP (single nucleotide polymorphism) rs7868992; $\left.p=1.85 \times 10^{-6}\right)[10,11]$.

Neben der Identifikation einzelner Polymorphismen diskutiert man gegenwärtig vor allem sogenannte seltene Varianten wie eine veränderte Anzahl bestimmter Genabschnitte (CNVs - copy number variants), die bei einigen Familien mit Tics identifiziert wurden, als möglicherweise ursächlich [12]. Weitere wichtige Impulse aus der genetischen Forschung kommen aus Untersuchungen, die störungsübergreifend gemeinsame Grundlagen von Tic- und Zwangsstörungen oder auch ADHS entschlüsseln wollen.

\section{Umwelteinflüsse}

Neben einem genetischen Einfluss werden verschiedene Umweltfaktoren als weitere Ursache diskutiert. Eine wichtige Hypothese bilden Veränderungen im angeborenen und erworbenen Immunsystem. In einer dänischen Kohortenstudie zeigte sich eine um $29 \%$ höhere Inzidenzrate für ein Tourette-Syndrom bei den Söhnen von Müttern mit einer Autoimmunerkrankung [13]. Die immunologischen Veränderungen bei Patienten mit Tics reichen von 
veränderten entzündlichen Zytokin-Konzentrationen über verstärkte Aktivierung von B- und Th1-Lymphozyten bis hin zu einer Aktivierung der Mikrogliazellen als Marker für eine Entzündungsreaktion im Gehirn.

Als mögliche Ursachen für die erhöhte Entzündungsreaktion bei Patienten mit Tics werden Infektionen mit bestimmten Erregern diskutiert. Allen voran könnte sich nach einer Infektion mit Typ A Streptokokken das Immunsystem - ähnlich wie bei der Chorea minorgegen körpereigene Strukturen wenden und so zu den Störungen in den Bewegungsabläufen führen. Das PANDAS-Syndrom wird in diesem Zusammenhang am häufigsten genannt und steht für: Pediatric Autoimmune Neuropsychiatric Disorders Associated with Streptococcal infections. Dieses Störungsbild wird in der Literatur sehr kontrovers diskutiert und wurde 2012 in das breiter gefasste PANS (Pediatric Acute Neuropsychiatric Syndromes) überführt, das auch andere Ursachen als Streptokokken-Infektionen umfasst.

Gegenwärtig wird eine großangelegte europaweite Studie (EMTICS) ausgewertet, die über das 7. Rahmenprogramm (FP7) der EU gefördert wurde, und das Zusammenspiel von genetischen Faktoren und Umwelteinflüssen (z. B. Infektionen) näher untersucht [14]. Es ist zu erwarten, dass die Ergebnisse dieser Studie einen wesentlichen Beitrag zur Aufklärung der fraglichen Beteiligung von Streptokokken-Infektionen bei der Genese von Tic-Störungen leisten.

Zusammengenommen kann das Tourette-Syndrom als komplexe neuropsychiatrische Erkrankung aufgefasst werden, die durch ein Zusammenspiel aus genetischen und Umweltfaktoren bedingt ist. In $>$ Tab. 1 sind die gegenwärtigen Ursachenmodelle nochmals zusammengefasst.

\section{Merke}

Das Tourette-Syndrom ist eine komplexe neuropsychiatrische Erkrankung. Es besteht eine Störung in kortiko-striato-pallido-thalamo-kortikalen Regelkreisen. Als Ursache wird ein komplexes Zusammenspiel aus genetischen und Umweltfaktoren vermutet.

\section{Klinische Zeichen und Verlauf}

Die Einteilung der Tics erfolgt nach Qualität (motorisch versus vokal) und Komplexität der Tics (einfach versus komplex, siehe $\triangleright$ Tab. 2). Die Zahl der im Einzelfall möglichen Kombinationen vokaler und motorischer Tics hinsichtlich ihrer Art, Anzahl, Frequenz, Intensität und Komplexität ist sehr hoch.

Häufig nehmen Tics bei Stress und Anspannung zu, während konzentrierte Tätigkeiten, Ruhe und Entspannung zu einer Reduktion der Tics beitragen können.
>Tab. 1 Ätiologie von Tic-Störungen

\begin{tabular}{|c|c|}
\hline Ursachenfaktor & Beobachtung \\
\hline Pathogenese & $\begin{array}{l}\text { - Komplexe Veränderung bestimmter } \\
\text { Hirnregionen } \\
\text { - Dysfunktion der kortiko-striato-pallido- } \\
\text { thalamo-kortikalen Regelkreise } \\
\text { - Veränderung im dopaminergen, seroto- } \\
\text { nergen, glutamatergen, GABAergen, } \\
\text { noradrenergen, cholinergen, histaminer- } \\
\text { gen, Cannabinoidrezeptor-System }\end{array}$ \\
\hline Genetik & $\begin{array}{l}\text { - Verwandte ersten Grades (Zwillings- und } \\
\text { Adoptionsstudien): Erkrankungsrisiko = 5- } \\
15 \% \\
\text { - Heritabilität 0,5 (zwischen 0,25 und } \\
0,77 \text { ) } \\
\text { - Polygenetische Ätiologie, bislang keine } \\
\text { genomweiten Signifikanzen }\end{array}$ \\
\hline Epigenetische Faktoren & $\begin{array}{l}\text { - Prä- und perinatale Komplikationen } \\
\text { - Immunologische Aspekte }\end{array}$ \\
\hline
\end{tabular}

\Tab.2 Einteilung von Tics nach Qualität und Komplexität mit Beispielen

\begin{tabular}{|c|c|c|}
\hline & motorisch & vokal \\
\hline einfach & $\begin{array}{l}\text { - Blinzeln } \\
\text { - Naserümpfen } \\
\text { - Augenrollen } \\
\text { - Nicken } \\
\text { - Schulterzucken }\end{array}$ & $\begin{array}{l}\text { - Räuspern } \\
\text { - Schniefen } \\
\text { - Schnüffeln } \\
\text {. Zischen } \\
\text { - Quieken } \\
\text { - Silben } \\
\text { - Tierlaute }\end{array}$ \\
\hline komplex & $\begin{array}{l}\text { - z. B. Verdrehen des } \\
\text { Oberkörpers bei gleich- } \\
\text { zeitigem Schulterzucken } \\
\text { - Kopropraxie (obszöne } \\
\text { Gesten) } \\
\text { - Echopraxie (Nachahmen } \\
\text { von Bewegungen } \\
\text { anderer) } \\
\text { - autoaggressive Tics }\end{array}$ & 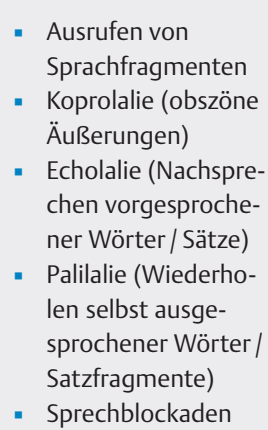 \\
\hline
\end{tabular}

Ein Großteil der Patienten berichtet von einem unangenehmen Vorgefühl (= „premonitory urge“), das sich nach Durchführung der Tics verbessert [4].

In der Regel beginnen Tic-Störungen im Alter von 67 Jahren [15]. Der Beginn ist langsam und im Verlauf wellenförmig, d.h. mit deutlichen Fluktuationen hinsichtlich Art und Häufigkeit der Tics („waxing and waning“). So kann es zu Beginn der Erkrankung 
lange Intervalle ohne Tics geben. In der Regel treten motorische Tics 2-3 Jahre früher auf als vokale Tics $[4,15]$. Tic-Störungen erreichen ihren Höhepunkt mit 10-11 Jahren. Bei vielen Kindern kommt es spontan zu einer deutlichen Reduktion der Tics im Jugendalter [4].

\section{Merke}

Ersterkrankungsalter 6. bis 7. Lebensjahr, Peak mit 1011 Jahren, oft deutliche Spontanreduktion im Jugendalter.

Die Kombination aus Tic-Beginn in der Kindheit, einem wellenförmigen Verlauf („waxing and waning“), Vorgefühl („premonitory urge“) und Unterdrückbarkeit von Tics unterscheiden diese von anderen Hyperkinesien (z. B. Myoklonien, Stereotypien).

\section{FALLBEISPIEL}

Der 8-jährige Patient kommt in Begleitung seiner Mutter zur Beratung in die Spezialambulanz für TicStörungen.

Tics bestünden seit dem 4. Lebensjahr und würden sich in Art und Häufigkeit abwechseln. Initial hätten nur motorische Tics bestanden, wie Schulterzucken, Augenrollen, Blinzeln und Bauchanspannen. Zwei Jahre später seien auch vokale Tics in Form von Hüsteln und „hm-hm“-artiger Geräusche hinzugetreten. Ein Vorgefühl in Form von „aufsteigendem Druck“ sei vorhanden und bessere sich auf Durchführung der Tics. Stress führe zu einer Zunahme von Tics, Musizieren zu einer Verbesserung. Der Vater des Patienten habe ebenfalls Tics, die gut unter Kontrolle seien und nur bei viel Stress in der Arbeit aufträten, eine Medikation werde nicht eingenommen. Hinweise auf psychiatrische Komorbiditäten finden sich nicht.

Eine ärztliche Vorstellung vor zwei Jahren mit körperlicher Untersuchung, Blutentnahme und EEG sei regelrecht gewesen.

Die Diagnose eines Tourette-Syndroms wird bestätigt und es erfolgt eine ausführliche Psychoedukation über Diagnose und Therapiemöglichkeiten. Eine Indikation zur medikamentösen Therapie besteht aktuell nicht.

\section{Diagnostik}

Entscheidend für die Diagnose einer Tic-Störung mit Abgrenzung zu Differentialdiagnosen ist die Anamnese zur Charakterisierung der Tics und deren typischer Verlauf (siehe Praxistipp unten und Abschnitt „Klinische Zeichen und Verlauf“) [16].

\section{PRAXISTIPP}

Typisch für Tic-Störungen sind:

- Beginn im Kindesalter

- Wellenförmiger Verlauf der Tics („,waxing and waning“): Fluktuationen hinsichtlich Art, Frequenz und Intensität

- Besserung der Tics durch Entspannung oder Konzentration

- Ticverstärkung durch Aufregung (positiv als auch negativ)

- Vorhandensein eines unangenehmen Vorgefühls („premonitory urge“) und Besserung nach Durchführung des Tics

- Unterdrückbarkeit des Tics für gewisse Zeit

Zur standardisierten Erhebung des Schweregrades der Tic-Störung kann man den Fragebogen Yale Global Tic Severity Scale (YGTSS) einsetzen. Dieser berücksichtigt motorische und vokale Tics hinsichtlich ihrer Anzahl, Frequenz, Intensität, Interferenz und Komplexität und bewertet auch die subjektive Belastung durch Tics [17]. Das Vorgefühl lässt sich mit Hilfe des Fragebogens Premonitory urge for tics scale (PUTS) erfassen [18], die Lebensqualität über die GTS-QoL (Gilles de la Tourette Syndrome-Quality of Life) Skala [19].

Neben der Erhebung eines psychopathologischen Befundes und einer neurologischen Untersuchung können ergänzend im Rahmen der Ausschlussdiagnostik Laboruntersuchungen (DD Morbus Wilson), EEG (DD Epilepsie) und cMRT (DD strukturelle Veränderungen der Basalganglien) veranlasst werden, eine Übersicht bietet Infobox 2 . Neben organisch bedingten Erkrankungen sind Tics differentialdiagnostisch auch von Stereotypien oder funktionellen Bewegungsstörungen abzugrenzen (siehe Infobox 3).

\section{INFOBOX 2: DAS GEHÖRT ZUR DIAGNOSTIK}

- Detaillierte Anamnese

- Beschreibung von Art, Häufigkeit, Intensität und Verteilung der Tics sowie funktioneller Einflussfaktoren (z. B. Stress), Vorgefühl („premonitory urge“) und Unterdrückbarkeit

- Beschreibung des Verlaufs (Erkrankungsalter, „waxing and waning“)

- Psychopathologischer Befund (inkl. möglicher assoziierter Störungen wie ADHS, Zwang, Depression, Angst, Autoaggression)

- Neurologischer Befund

- Labor (inkl. Kupfer-Stoffwechsel)

- Ggf. EEG und cMRT

- Erfassung des Schweregrads:

- Yale Global Tic Severity Scale (YGTSS)

- Erfassung des Vorgefühls:

- Premonitory Urge for Tics Scale (PUTS) 
INFOBOX 3: DIFFERENTIALDIAGNOSEN DER

TIC-STÖRUNG

- Dystonie

- Myoklonien

- Substanz-induzierte Dyskinesie

- Chorea Huntington

- Chorea Sydenham

- Morbus Wilson

- Neuroakanthozytose

- Psychogene Bewegungsstörung

- Stereotypien

\section{Komorbiditäten}

85-90\% der Patienten mit Tic-Störung haben komorbide psychiatrische Symptome oder Erkrankungen. Die häufigsten sind [5]:

- ADHS

- Zwangsstörung/-symptome

- Depression

- Angststörung

Merke

Die Erhebung eines psychopathologischen Befundes bei Verdacht auf eine Tic-Störung sollte man aufgrund häufiger psychiatrischer Komorbiditäten nicht vergessen!

\section{Behandlungsbedarf}

Patienten mit Tic-Störungen benötigen nicht zwingend eine Therapie. Über $90 \%$ der Betroffenen erleben eine Spontanreduktion der Tics im Jugendalter, weshalb erst bei Patienten mit schweren Tics und deutlichen Einschränkungen oder erheblichen psychosozialen Belastungen eine Behandlung durchgeführt werden sollte [4, 20]. Oft liegen zwischen dem ersten Auftreten der Tics und einer korrekten Diagnosestellung mehrere Jahre. Ein Großteil der Patienten ist daher bereits durch eine korrekte Diagnose entlastet. Eine Heilung der Erkrankung ist im Moment nicht möglich [20]. Wichtiger Bestandteil der Behandlung ist eine ausführliche Psychoedukation der Betroffenen selbst sowie des sozialen Umfelds. Es sollte auf Symptome, Verlauf, Fluktuationen, Prognose, Ursachen und Behandlungsmöglichkeiten eingegangen werden [4]. Insbesondere Kinder können dadurch die Erkrankung besser annehmen und akzeptieren. Mobbing und Stigmatisierung können reduziert werden $[4,20]$.

\section{Selbsthilfegruppen}

Im Rahmen der Psychoedukation sollte auch die Möglichkeit einer Beratung durch Selbsthilfegruppen erwähnt werden. Diese bieten eine gute Möglichkeit mit anderen Betroffenen in Kontakt zu kommen und sich gegenseitig auszutauschen. Die Tourette-Gesellschaft Deutschland e. V. (TGD; https: / /tourette-gesellschaft.de) ist beispielsweise deutschlandweit verteilt und setzt sich für eine Aufklärung der Öffentlichkeit über die Erkrankung sowie eine Verbesserung der Behandlungsmöglichkeit ein. Ebenso ist der Interessen-Verband Tic \& Tourette Syndrom (IVTS; htt ps: //iv-ts.de) darum bemüht, das Leben der Betroffenen einfacher zu gestalten. Es werden Beratungen, Seminare sowie Informationsmaterial angeboten [4].

\section{Pharmakotherapie}

Wie bereits erwähnt, muss nicht jeder Patient mit einer Tic-Störung behandelt werden. In vielen Fällen führen bereits eine korrekte Diagnosestellung und Psychoedukation zu einer deutlichen Entlastung. Praxistipp 2 fasst die Indikationen für eine medikamentöse Therapie zusammen.

\section{PRAXISTIPP}

Indikationen für eine Pharmakotherapie bei Tic-

Störungen liegen vor:

- Wenn Tics körperliche Beschwerden bereiten (z. B. Schmerzen, Verletzungen).

- Wenn es zu anhaltenden psychosozialen Belastungsfaktoren kommt (z. B. Mobbing, soziale Isolation).

- Wenn sekundäre psychische Probleme zustande kommen (z. B. Depression, Angststörung).

- Wenn die Leistungsfähigkeit deutlich abnimmt (z. B. in Schule oder Arbeit).

- Bei hoher subjektiver Belastung durch Tics.

- Falls die Psychotherapie nicht ausreichend wirksam ist.

Zur psychopharmakologischen Behandlung von TicStörungen und dem Tourette-Syndrom stehen mittlerweile eine Reihe wirksamer Behandlungsoptionen zur Verfügung. Eine Schwierigkeit im klinischen Alltag bleibt allerdings der überwiegende off-label Status der meisten Präparate. Im europäischen Raum steht seit 2011 die Leitlinie der Europäischen TouretteGesellschaft zur Verfügung [21]. Neuere Entwicklungen seit Erscheinen dieser Leitlinie werden natürlich nicht berücksichtigt, sodass sich im klinischen Alltag bereits eine etwas modifizierte Vorgehensweise etabliert hat.

Den angenommenen Pathomechanismen von Tic-Störungen entsprechend greifen die meisten verwendeten Psychopharmaka ins dopaminerge System ein, als einem der wesentlichen Neurotransmittersysteme der kortikostriato-pallido-thalamo-kortikalen Regelkreise. Daneben werden Präparate verwendet, die auf das noradrenerge, GABAerge oder Cannabinoid-System Einfluss nehmen [9]. 
In Tabelle 3 sind die Medikamente aufgelistet, die bei uns am häufigsten zum Einsatz kommen, mit ihrem pharmakologischen Wirkansatz, einer Dosisempfehlung für Erwachsene und dem Zulassungsstatus.

Einer neueren Übersichtsarbeit zufolge bestehen in der Beurteilung der Rangfolge der medikamentösen Therapieoptionen zwischen den USA und anderen Ländern deutliche Unterschiede. Quezada und Coffman sehen auch in psychotherapeutischen Verfahren die erste Wahl, die als Basistherapie alle weiteren Interventionen begleiten sollte [22]. Als 2. Wahl werden die noradrenerg wirksamen Medikamente Clonidin und Guanfacin in den USA und die Gruppe der Benzamide außerhalb der USA eingeteilt. Danach folgen der GABA-B-Agonist Baclofen (USA), die VMATInhibitoren Tetrabenazin und Deutetrabenazin sowie das Antikonvulsivum Topiramat (andere Länder). Erst in letzter Folge werden die neueren und klassischen Antipsychotika erwähnt.
Dies ist vor allem auf das mitunter erhebliche Nebenwirkungspotential dieser Medikamentengruppe zurückzuführen.

- Bei den Antipsychotika der 2. Generation (SGA; second generation antipsychotics; atypische Antipsychotika) sind dies insbesondere metabolische Auffälligkeiten wie eine medikamenteninduzierte Gewichtszunahme oder Verschlechterung der Nüchtern-Blutfette und des Nüchtern-Blutzuckers.

- Gegen die Vertreter der 1. Generation (FGA; first generation antipsychotics; klassische Antipsychotika) spricht weiterhin das Risiko zur Entwicklung extrapyramidal-motorischer Symptome bis hin zu Spätdyskinesien in seltenen Fällen.

In unserer Tic-Sprechstunde lehnen wir uns mehr an die Empfehlungen der ESSTS-Leitlinie an und setzen SGA deutlich früher ein. Aufgrund der guten Verträglichkeit haben wir daher in > Tab. 3 Tiaprid und Aripiprazol als Mittel der ersten Wahl eingeordnet, aufgrund der sehr

-Tab. 3 Pharmakotherapie - Überblick

\begin{tabular}{|c|c|c|c|c|c|}
\hline Präparat & Medikamenten-klasse & Wirkmechanismus $^{1}$ & Dosisempfehlung ${ }^{2}$ & Zulassung $^{3}$ & Einsat $z^{4}$ \\
\hline Aripiprazol & SGA & $\begin{array}{l}\text { Partieller D2 und 5HT1A } \\
\text { Agonismus }\end{array}$ & $2,5-15 \mathrm{mg}$ & Off-label (in USA on-label) & 1. Wahl \\
\hline Risperidon & SGA & D2-, 5HT2A-Blockade & $1-4 \mathrm{mg}$ & Off-label & 2. Wahl \\
\hline Olanzapin & SGA & D2-, 5HT2A-Blockade & $2,5-10 \mathrm{mg}$ & Off-label & 3. Wahl \\
\hline Quetiapin & SGA & D2-Blockade & $25-300 \mathrm{mg}$ & Off-label & 3. Wahl \\
\hline Ziprasidon & SGA & D2-, 5HT2A-Blockade & $20-60 \mathrm{mg}$ & Off-label & 3. Wahl \\
\hline Haloperidol & FGA & D2-Blockade & $1-4 \mathrm{mg}$ & On-label & 3. Wahl \\
\hline Pimozid & FGA & D2-Blockade & $1-4 \mathrm{mg}$ & Off-label (in USA on-label) & 3. Wahl \\
\hline Amisulprid & Benzamid & D2 / D3-Blockade & $50-600 \mathrm{mg}$ & Off-label & 3. Wahl \\
\hline Sulpirid & Benzamid & D2-Blockade & $50-400 \mathrm{mg}$ & Off-label & 3. Wahl \\
\hline Tiaprid & Benzamid & D2 / D3-Blockade & $150-600 \mathrm{mg}$ & Off-label & 1. Wahl \\
\hline Tetrabenazin & Vesikelentleerer & VMAT2-Inhibitor & $25-150 \mathrm{mg}$ & Off-label & 4. Wahl \\
\hline Clonidin & Antihypertonikum & Zentraler a2-Agonist & $75-300 \mu \mathrm{g}$ & Off-label & 2. Wahl \\
\hline Nabiximols & $\begin{array}{l}\text { Cannabinoid (CBD: } \\
\text { THC }-1: 1)\end{array}$ & $\begin{array}{l}\text { Partieller CB1- / CB2- } \\
\text { Agonist }\end{array}$ & 2-12 Sprühstöße & Off-label & 3. Wahl \\
\hline Dronabinol & Cannabinoid (THC) & $\begin{array}{l}\text { Partieller CB1- / CB2- } \\
\text { Agonist }\end{array}$ & $5-10 \mathrm{mg}$ & Off-label & 3. Wahl \\
\hline
\end{tabular}

1 Nur postulierte Hauptmechanismen, darüber hinaus bestehen meist Affinitäten zu weiteren Rezeptorklassen.

2 Die Dosisempfehlungen richten sich nach den Dosierungen, die in der Tic-Sprechstunde der Psychiatrischen Klinik des Klinikums der Universität München üblicherweise zum Einsatz kommen. Diese können von den Dosierungsempfehlungen nach Fachinformation in den zugelassenen Indikationen abweichen. 3 Der Zulassungsstatus bezieht sich auf Deutschland, in Klammern sind teilweise bekannte Zulassungen in anderen Ländern vermerkt, weitere Zulassungen können in anderen Ländern bestehen, die von den Autoren nicht recherchiert wurden.

4 Die Empfehlungen entsprechen dem klinischen Gebrauch in der Tic-Sprechstunde der Psychiatrischen Klinik des Klinikums der Universität München, im Text sind die Empfehlungen von Leitlinien aufgeführt.

SGA: second generation antipsychotic (atypisches Antipsychotikum); FGA: first generation antipsychotic (typisches Antipsychotikum/ Neuroleptikum); VMAT: vesikulärer Monoamintransporter; CBD: Cannabidiol; THC: Tetrahydrocannabinol; CB1 / CB2: Cannabinoid-Rezeptor 1/2; D2: Dopamin Typ 2 Rezeptor; D3: Dopamin Typ 3 Rezeptor; 5HT1A: Serotonin Typ 1A Rezeptor; 5HT2A: Serotonin Typ 2A Rezeptor; $\alpha 2$ : noradrenerger $\alpha 2$ - Rezeptor. 
guten klinischen Wirksamkeit Risperidon als 2. Wahlebenso wie Clonidin, das bei guter Verträglichkeit oft als Bedarfsmedikament zur Milderung von Tics unter erhöhter Stressbelastung Einsatz findet. Alle anderen Präparate, bis auf Tetrabenazin, haben wir als Mittel der 3. Wahl eingeordnet, nachdem entsprechende Vergleichsstudien fehlen und es dann in der Regel unter Abwägung potentieller Nebenwirkungen in einem partizipativen Prozess mit dem Patienten zur Auswahl kommt. Tetrabenazin zeigte sich bei den wenigen Anwendungen in unserer Ambulanz meist als nicht ausreichend gut verträglich.

\section{Merke}

Die Pharmakotherapie von Tic-Störungen setzt insbesondere an den Neurotransmittersystemen von Dopamin und Noradrenalin an. In der Behandlung mit atypischen Antipsychotika ist auf die Entwicklung metabolischer Nebenwirkungen zu achten.

\section{FALLBEISPIEL - FORTSETZUNG}

Tics nahmen im Verlauf zu und konnten nicht ausreichend durch progressive Muskelrelaxation kontrolliert werden. Eine Tic-spezifische ambulante Verhaltenstherapie war nicht verfügbar. Bei zunehmender Belastung erfolgte im 10. Lebensjahr eine Medikation mit Tiaprid bis $200 \mathrm{mg} / \mathrm{d}$. Hierunter besserten sich Tics nur initial, sodass bei zusätzlichem Prolaktinanstieg eine Umstellung von Tiaprid auf Aripiprazol erfolgte. Aripiprazol wurde bis $15 \mathrm{mg} / \mathrm{d}$ aufdosiert, gut vertragen und führte zu einer deutlichen Besserung der Tics.

\section{Cannabinoide}

In den letzten Jahren hat sich-zumindest in unserer Tic-Sprechstunde-zunehmend ein Trend entwickelt, frühzeitig in der Pharmakotherapie auch Cannabisprodukte einzusetzen. Das hat mehrere Gründe: Die Gesetzeslage zur Verschreibung von Cannabinoiden ist vereinfacht worden, die Kassen übernehmen häufiger die Kosten für diese Therapieform, es werden vermehrt qualitativ hochwertige Studien in dieser Substanzgruppe durchgeführt, die medikamentösen Alternativen haben oft erhebliche Nebenwirkungen und die Patienten wünschen sich vermehrt einen Therapieversuch mit Cannabinoiden.

Zur Verfügung stehen

- der Wirkstoff Tetrahydrocannabinol (THC) als Dronabinol-Tropfen oder -Kapseln,

- THC und der Wirkstoff Cannabidiol (CBD) im Verhältnis 1:1 als Mundspray (Sativex; Wirkstoff Nabiximols) sowie

- Cannabisblüten mit sehr unterschiedlichen Verhältnissen von THC und CBD.
Noch ist unklar, welches Konzentrationsverhältnis von THC und CBD für welche Patienten oder Symptomkonstellationen am besten wirksam ist. Im klinischen Praxisalltag ist ein weiteres Hindernis die sehr stark schwankende Verfügbarkeit einzelner Cannabisblüten, sodass die Patienten immer wieder auf ein anderes Präparat ausweichen müssen.

Aber auch Cannabinoide haben natürlich Nebenwirkungen wie z. B. Müdigkeit, Schwindel, trockener Mund und eine Reihe psychischer Veränderungen (z. B. Angstsymptome) [23]. Daneben ist ein gesteigertes Risiko, eine Psychose zu entwickeln, vor allem im Jugendalter zu beachten.

Zuletzt werden Cannabisblüten in der Regel inhalativ eingenommen, sodass die negativen Auswirkungen des Rauchens ein zusätzliches Gesundheitsrisiko darstellen. Aus diesen Gründen und aufgrund der bislang noch geringen Datenlage sollten Cannabinoide erst nach einem Therapieversuch mit einem Mittel der ersten Wahl (siehe $\triangleright$ Tab. 3) eingesetzt werden.

Gegenwärtig laufen in Deutschland und international mehrere Studien, welche die Wirksamkeit von oralen Therapieformen, die in das Cannabinoid-System einwirken, untersuchen. Es besteht also Hoffnung, dass das Therapiearsenal in der Zukunft in diesem Bereich deutlich erweitert wird, ohne die negativen Auswirkungen von Rauchen in Kauf nehmen zu müssen.

\section{Weitere Therapiemöglichkeiten}

In $\triangleright$ Tab. 3 nicht aufgeführte Medikamente umfassen auf den Botenstoff GABA wirksame Substanzen wie das Benzodiazepin Clonazepam (CAVE: Toleranzentwicklung), der GABA-B-Agonist Baclofen (nur sehr geringe Evidenz) oder das Antikonvulsivum Topiramat (geringe Evidenz), die alle in der Regel erst eingesetzt werden, wenn andere Therapieoptionen erfolglos bleiben.

Eine Möglichkeit, lokal umschriebene motorische Tics zu behandeln, ist die Injektion von Botulinumtoxin.

Bei komorbiden psychiatrischen Störungsbildern sollten bestimmte Substanzen aus $>$ Tab. 3 bevorzugt werden: Bei komorbiden Zwangsstörungen oder -symptomen zeigen sich Aripiprazol oder Risperidon häufig erfolgreich. Die Therapie mit Antidepressiva wie der Gruppe der SSRIs (Selektive Serotonin-Rückaufnahme-Inhibitoren) oder dem Trizyklikum Clomipramin stellt ebenfalls eine sinnvolle Ergänzung dar, zeigt sich aber im Vergleich zur Behandlung von Zwängen ohne begleitende Tics oft weniger hilfreich.

Bei komorbider ADHS können sich die noradrenerg wirksamen Substanzen Clonidin und Guanfacin sowohl auf die Tics als auch die ADHS-typischen 
Symptome positiv auswirken. Eine Behandlung mit Methylphenidat bleibt aber die erste Wahl und führt statistisch auch nicht zu einer Zunahme der Tics. Eine Alternative wäre Atomoxetin sollte sich im Einzelfall eine Verschlechterung der Tics unter Methylphenidat zeigen.

\section{Merke}

Zur Pharmakotherapie komorbider Zwänge können SSRIs, Clomipramin und als Alternativen Aripiprazol oder Risperidon eingesetzt werden. Bei komorbider ADHS ist Methylphenidat die erste Wahl. Alternativen sind Atomoxetin, Clonidin und Guanfacin.

Neben den bislang genannten - primär auf die Symptomatik abzielenden Therapieformen - ist eine Behandlung mit Antibiotika oder intravenöser Immunglobulingabe eine in der Theorie ursächliche Behandlungsform. Beide wurden unter der Vorstellung der Beteiligung von Infektionen (Streptokokkeninfekte bei PANDAS) bzw. Veränderungen im Immunsystem (erhöhte zirkulierende Antikörper) eingesetzt. Der Auffassung der Mehrzahl der europäischen Experten nach sollte eine solche Therapie jedoch ausschließlich im Rahmen von Studien erfolgen. Die weiter oben erwähnte EMTICS-Studie wird hier in naher Zukunft hoffentlich zu mehr Klarheit und neuen Empfehlungen führen [14].

\section{Möglichkeiten bei Therapieresistenz}

Bei Therapieresistenz steht mit der Tiefenhirnstimulation (THS) eine mittlerweile in einigen spezialisierten Zentren angebotene Therapiealternative zur Verfügung [24].

Bei diesem Verfahren werden im Rahmen einer stereotaktischen neurochirurgischen Operation Elektroden in die betroffenen Hirnareale implantiert und über einen im Brust- oder Bauchbereich eingesetzten Impulsgeber, der gleichzeitig als Batterie dient, gesteuert. Durch die Stimulation sollen die gestörten kortiko-striato-pallido-thalamo-kortikalen Regelkreise positiv beeinflusst werden. Dieser grundlegenden pathophysiologischen Annahme zur Entstehung von Tics nach kommen entsprechend verschiedene Hirnareale als Stimulationsort in Frage: Am häufigsten werden Bereiche im Thalamus oder im Globus pallidus internus als Zielort gewählt.

Wie bei jedem invasiven Eingriff können Komplikationen wie Blutungen oder Infektionen auftreten, diese liegen je nach Zentrum jedoch bei unter $1 \%$. Mehrere Studien ergaben, dass die THS-OP die Lebensqualität von Patienten mit Tourette-Syndrom verbessern kann [25, 26]. Die Indikation zur Durchführung sollte nur in spezialisierten Zentren und unter enger Zusammenarbeit zwischen behandelnden Psychiatern/Neurologen und Neurochirurgen erfolgen.
Als mögliche Alternativen zu einer THS werden gegenwärtig neuere und nicht-invasive Hirnstimulationsverfahren, wie die transkranielle Gleichstromstimulation, auf ihre Wirksamkeit bei Tic-Störungen untersucht. Noch gibt es zu wenige Daten zu dieser risikoärmeren Behandlungsform, um einen häufigeren Einsatz zu rechtfertigen.

\section{Psychotherapie}

Für Tic-Störungen gibt es diverse Therapiemöglichkeiten. Nach den Europäischen Leitlinien ist die Verhaltenstherapie die Behandlungsmethode der ersten Wahl [27]. Hierunter fallen

- die Exposure and Response Prevention (ERP),

- das Habit Reversal Training (HRT) sowie

- die Comprehensive Behavioral Intervention for Tics (CBIT).

Die ERP, das HRT und die CBIT werden als ambulante Kurzzeittherapien im Rahmen von 8 Sitzungen angeboten (in 10 Wochen mit 2 fakultativen Booster-Sitzungen). Nebenwirkungen sind nicht zu erwarten [4].

\section{Merke}

Die Verhaltenstherapie ist nach den Europäischen Leitlinien Methode der ersten Wahl.

\section{ERP}

Die Methode ERP unterbricht die Abfolge von Vorgefühl und Tic. Der Patient lernt die Tics gezielt zu unterdrücken. Dadurch kommt es als erstes zu einer Habituation, im Anschluss daran auch zu einer Reduktion des Vorgefühls und der Tics. Die ERP eignet sich insbesondere, wenn jemand unter vielen verschiedenen Tics leidet. Ebenso ist sie bei starken Vorgefühlen geeignet [4].

\section{HRT}

Das HRT wendet man vor allem bei automatisierten und situationsspezifischen Tics an. Die Methode beinhaltet zunächst ein Wahrnehmungstraining: Der Patient soll lernen, seine unerwünschten Verhaltensgewohnheiten bewusst wahrzunehmen und auslösende und aufrechterhaltende Faktoren zu erkennen. Ebenso soll er das Vorgefühl bewusst wahrnehmen. Patienten, die nicht von Vorgefühlen berichten, werden dazu angehalten, auf mögliche andere Körpersignale zu achten, die einem Tic vorausgehen, zum Beispiel Gefühle oder Handlungen. Darauf folgen ein Training zum Umgang mit unvorhergesehenen Ereignissen sowie ein Entspannungstraining.

Das Kernelement des HRT ist das Identifizieren und Erlernen eines Alternativverhaltens. Verhaltensweisen, die mit der Ausführung des Tics inkompatibel sind, werden erarbeitet und geübt (=Gegenbewegung bzw. „Konkurrenzreaktion“). Nehmen wir zum Beispiel einen Patienten, der einen Tic hat, der beinhaltet, die Schulter hochzuziehen. Eine mögliche Gegenbewegung wäre hier 
-Tab.4 Konkurrenzreaktionen, die in HRT/CBIT geübt werden (nach [4])

\begin{tabular}{|l|l|}
\hline Tic & Konkurrenzreaktion \\
\hline Motorische Tics & \\
\hline Blinzeln & $\begin{array}{l}\text { Die Augen weit offen und den } \\
\text { Blick auf einen Punkt fixiert halten. }\end{array}$ \\
\hline Kopfschütteln & $\begin{array}{l}\text { Kopf durch Anspannen der } \\
\text { Nackenmuskulatur stillhalten. }\end{array}$ \\
\hline Mundaufreißen & $\begin{array}{l}\text { Lippen zusammenpressen; ggf. } \\
\text { zusätzlich Zähne } \\
\text { zusammenbeißen. }\end{array}$ \\
\hline Mittelfinger zeigen & $\begin{array}{l}\text { Hand zur Faust ballen; ggf. zusätz- } \\
\text { lich Arm an die Körperseite } \\
\text { drücken. }\end{array}$ \\
\hline Vokale Tics & $\begin{array}{l}\text { Langsam und ohne Pause durch } \\
\text { die Nase ein- und ausatmen. }\end{array}$ \\
\hline Schniefen & $\begin{array}{l}\text { Langsam und ohne Pause durch } \\
\text { die Nase ein- und ausatmen; die } \\
\text { Lippen fest aufeinander pressen. }\end{array}$ \\
\hline Fluchen / & \\
\hline Beschimpfen & \\
\hline
\end{tabular}

ein Herunterdrücken der Schulter. $>$ Tab. 4 zeigt weitere Beispiele. Der Patient wird dabei durch den Therapeuten immer wieder zur Verhaltensänderung motiviert und positiv verstärkt. Der letzte Baustein des HRT ist die Automatisierung und Generalisierung des Verhaltens. Der Patient übt, die neuen Verhaltensweisen im Alltag anstatt der Tics anzuwenden [4]. Infobox 4 fasst die zentralen Elemente des HRT zusammen.

INFOBOX 4: HABIT REVERSAL TRAI-

NING (HRT)

- Wahrnehmungstraining

- Training im Umgang mit unvorhergesehenen Ereignissen

- Entspannungstraining

- Identifizieren und Erlernen eines Alternativverhaltens („Konkurrenzreaktion“)

- Automatisierung und Generalisierung des Verhaltens

\section{CBIT}

Das Verfahren CBIT erweitert das HRT um eine detaillierte Psychoedukation, ein Achtsamkeitstraining und Funktionsanalysen [4].

\section{Wirksamkeit}

Studien zeigen, dass die Methoden HRT und CBIT zu einer Tic-Reduktion von bis zu 30-40\% führen können. Beide Verfahren sind sowohl für Kinder und Jugendliche als auch für Erwachsene mit Tic-Störungen effektive Behandlungsmethoden [28]. Die Verfahren HRT und
CBIT haben gegenüber der ERP den Vorteil, dass eine Behandlung mit ihnen auch möglich ist, wenn während der Therapiesitzung keine Tics vorliegen. So können beispielsweise Gegenbewegungen erarbeitet werden, die der Patient zu Hause einüben kann [4].

\section{Merke}

Durch Verhaltenstherapie lassen sich Tics um bis zu 30$40 \%$ reduzieren.

\section{Therapeutenmangel}

Aktuell ist es leider so, dass es in Deutschland unserer Erfahrung nach sehr wenige qualifizierte Therapeuten gibt, die Tic-Störungen behandeln. Hinzu kommt, dass die Wartezeiten auf einen ambulanten Psychotherapieplatz mehrere Monate betragen können. Daher können nur wenige Patienten CBIT/HRT oder ERP in Anspruch nehmen [4]. Zurzeit ermittelt eine multizentrische deutschlandweite Studie die Wirksamkeit eines ONLINETherapieverfahrens. Hierbei werden insbesondere Gegenbewegungen eingeübt, d. h. das CBIT kommt zum Einsatz [29].

\section{FALLBEISPIEL - FORTSETZUNG}

Im 13. Lebensjahr bestanden motorische Tics vorrangig unter Stress, z. B. bei Schulaufgaben. Bei zunehmender Bedeutung seiner Außenwirkung kam es bei dem Patienten zudem zu einer wachsenden emotionalen Anspannung durch das Auftreten von Tics im sozialen Kontext.

Um eine bessere Kontrolle über Tics zu erlangen, wurde eine ambulante Verhaltenstherapie begonnen. Diese konzentriert sich auf das Erlernen von HRT. Unterstützend kommen Achtsamkeitsübungen und Entspannungsübungen zum Einsatz, bereits vorhandene Ressourcen und Selbstbewusstsein werden gefördert. Die Medikation blieb unverändert. Leistungen auf dem Gymnasium seien zufriedenstellend und er sei sozial gut integriert.

\section{KERNAUSSAGEN}

- Tics beginnen meist im Kindesalter und zeigen einen fluktuierenden Verlauf

- Unterschieden werden vorübergehende Tic-Störungen (<12 Monate) von chronischen Tic-Störungen ( $\geq 12$ Monate)

- Das Tourette-Syndrom ist eine chronische Tic-Störung und weist motorische und mindestens einen vokalen Tic auf

- Pathophysiologisch bestehen Störungen in kortikostriato-pallido-thalamo-kortikalen Regelkreisen

- Ursächlich werden komplexe Gen-Umwelt-Interaktionen vermutet 
- Psychiatrische Komorbiditäten sollten bei Diagnose und Therapie von Tic-Störungen berücksichtigt werden

- Diagnostik und Psychoedukation führen meist zu einer deutlichen Entlastung bei Betroffenen mit Tic-Störungen und deren Angehörigen

- Verhaltenstherapie ist die Therapie der ersten Wahl; zur Auswahl stehen HRT, ERP und CBIT

- In Deutschland ist nur Haloperidol zur Behandlung von Tic-Störungen zugelassen; aufgrund eines besseren Nebenwirkungsspektrums stellen jedoch Aripiprazol oder Tiaprid die erste Wahl bei einer medikamentösen Therapie dar (off-label)

- Ziel ist eine gute Lebensqualität und nicht Symptomkontrolle um jeden Preis

\section{Wissenschaftlich verantwortlich gemäß Zertifizierungsbestimmungen}

Wissenschaftlich verantwortlich gemäß Zertifizierungsbestimmungen für diesen Beitrag ist Dr. med. Richard Musil.

\section{Interessenkonflikt}

Dr. Richard Musil hat Vortragshonorare von Otsuka/Lundbeck erhalten. Die Autoren sind Mitglied in der Europäischen Tourette-Gesellschaft (ESSTS) und sind an klinischen Multicenterstudien im Bereich Tourette beteiligt, die von öffentlichen Trägern (DFG, BMBF, EU) oder pharmazeutischen Unternehmen gefördert werden.

\section{Autorinnen / Autoren}

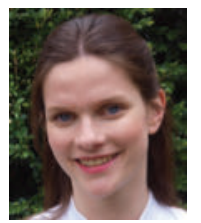

\section{Sc. Jaana M. L. Schnell}

Psychologin (M.Sc.), Jahrgang 1990. 20112016 Studium der Psychologie an der LMU München. Seit 2016 Promotionsstudium bei Prof. Dr. Dipl.-Psych. Norbert Müller (LMU; TicStörungen / Psychoneuroimmunologie). Seit 2017 Ausbildung zur Psychologischen Psychotherapeutin (VT) am CIP München und wissenschaftliche Mitarbeiterin in der Arbeitsgruppe Tourette-Syndrom (LMU). Schwerpunkte: Ticstörungen, Depressionen. E-Mail: Jaana.schnel !@med.uni-muenchen.de

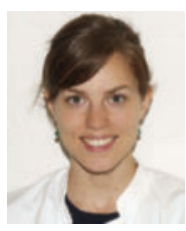

\section{Dr. med. Elif Weidinger}

Fachärztin für Psychiatrie und Psychotherapie, Jahrgang 1983. Studium der Humanmedizin an der LMU München 2002-2009, Promotion zum Thema „Antikörpertiter bei Tourette-Syndrom und Schizophrenie“. Tätig in der Institutsambulanz der Psychiatrischen Klinik der LMU München. Schwerpunkte: Ticstörungen, Depressionen. E-Mail: Elif.weidinger@med.unimuenchen.de

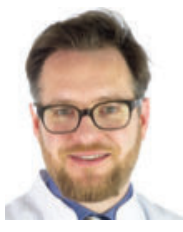

\section{Dr. med. Richard Musil}

Facharzt für Psychiatrie und Psychotherapie, Jahrgang 1977. Oberarzt an der Klinik für Psychiatrie und Psychotherapie des Klinikums der Universität München (Schwerpunktstation für Borderline Persönlichkeitsstörungen und Bipolare Störungen, Leitung der Tourette-Ambulanz). Studium der Humanmedizin an der TU München 1997-2004. Schwerpunkte: Ticstörungen, Borderline-Persönlichkeitsstörung, Pharmakogenetik, Akupunktur in der Psychiatrie. E-Mail: Richard.musil@med.uni-muenchen. de

\section{Korrespondenzadresse}

\section{Dr. med. Richard Musil}

Klinik für Psychiatrie und Psychotherapie des Klinikums der Universität München

Nußbaumstr. 7

80336 München

E-Mail: Richard.musil@med.uni-muenchen.de

\section{Literatur}

[1] Schnell JML, Weidinger E, Musil R. Patienten mit Tics. MMW Fortschritte der Medizin 2018; 160: 47-50

[2] Dilling $\mathrm{H}$, Mombour W, Schmidt $\mathrm{MH}$ et al. Internationale Klassifikation psychischer Störungen: ICD-10, Kapitel V (F, klinisch-diagnostische Leitlinien. 1991

[3] Robertson MM. The prevalence and epidemiology of Gilles de la Tourette syndrome: Part 1: The epidemiological and prevalence studies. Journal of psychosomatic research 2008; 65: 461-472

[4] Müller-Vahl K. Tourette-Syndrom und andere Tic-Erkrankungen im Kindes- und Erwachsenenalter. Berlin: Medizinisch Wissenschaftliche Verlagsgesellschaft; 2014

[5] Robertson MM. A personal 35 year perspective on Gilles de la Tourette syndrome: prevalence, phenomenology, comorbidities, and coexistent psychopathologies. The Lancet Psychiatry 2015; 2: 68-87

[6] Martino D, Leckman JF. Tourette syndrome: Oxford University Press; 2013

[7] Worbe $Y$, Marrakchi-Kacem L, Lecomte $S$ et al. Altered structural connectivity of cortico-striato-pallido-thalamic networks in Gilles de la Tourette syndrome. Brain: a journal of neurology 2015; 138: 472-482

[8] Conceicao VA, Dias A, Farinha AC et al. Premonitory urges and tics in Tourette syndrome: computational mechanisms and neural correlates. Current opinion in neurobiology 2017; 46: 187-199

[9] Robertson MM, Eapen V, Singer HS et al. Gilles de la Tourette syndrome. Nature reviews Disease primers 2017; 3: 16097

[10] Paschou P, Yu D, Gerber G et al. Genetic association signal near NTN 4 in T ourette syndrome. Annals of neurology 2014; 76: $310-315$

[11] Qi Y, Zheng Y, Li Z et al. Progress in Genetic Studies of Tourette's Syndrome. Brain sciences 2017; 7

[12] Huang AY, Yu D, Davis LK et al. Rare copy number variants in NRXN1 and CNTN6 increase risk for Tourette syndrome. Neuron 2017; 94: 1101-1111 
[13] Dalsgaard S, Waltoft BL, Leckman JF et al. Maternal history of autoimmune disease and later development of tourette syndrome in offspring. Journal of the American Academy of Child \& Adolescent Psychiatry 2015; 54: 495-501

[14] Schrag A, Martino D, Apter A et al. European Multicentre Tics in Children Studies (EMTICS): protocol for two cohort studies to assess risk factors for tic onset and exacerbation in children and adolescents. European child \& adolescent psychiatry 2018. 1-19

[15] Freeman RD, Fast DK, Burd L et al. An international perspective on Tourette syndrome: selected findings from 3500 individuals in 22 countries. Developmental medicine and child neurology 2000; 42: 436-447

[16] Stern JS. Tourette's syndrome and its borderland. Practical neurology 2018; 18: 262-270

[17] Leckman JF, Riddle MA, Hardin MT et al. The Yale Global Tic Severity Scale: initial testing of a clinician-rated scale of tic severity. Journal of the American Academy of Child \& Adolescent Psychiatry 1989; 28: 566-573

[18] Woods DW, Piacentini J, Himle MB et al. Premonitory Urge for Tics Scale (PUTS): initial psychometric results and examination of the premonitory urge phenomenon in youths with Tic disorders. Journal of Developmental \& Behavioral Pediatrics 2005; 26: 397-403

[19] Cavanna A, Schrag A, Morley D et al. The Gilles de la Tourette syndrome - Quality of Life Scale (GTS-QOL) development and validation. Neurology 2008; 71: 1410-1416

[20] Ludolph AG, Roessner V, Münchau A et al. Tourette syndrome and other tic disorders in childhood, adolescence and adulthood. Deutsches Ärzteblatt International 2012; 109: 821

[21] Roessner V, Plessen K], Rothenberger A et al. European clinical guidelines for Tourette syndrome and other tic disorders. Part II: pharmacological treatment. European child \& adolescent psychiatry 2011; 20: 173-196

[22] Quezada J, Coffman KA. Current approaches and new developments in the pharmacological management of Tourette syndrome. CNS drugs 2018. 1-13
[23] Müller-Vahl KR. Treatment of Tourette syndrome with cannabinoids. Behavioural neurology 2013; 27: 119-124

[24] Müller-Vahl KR, Cath DC, Cavanna AE et al. European clinical guidelines for Tourette syndrome and other tic disorders. Part IV: deep brain stimulation. European child \& adolescent psychiatry 2011; 20: 209-217

[25] Martinez-Ramirez D, Jimenez-Shahed J, Leckman JF et al. Efficacy and safety of deep brain stimulation in Tourette syndrome: the international Tourette syndrome deep brain stimulation public database and registry. JAMA neurology 2018; 75: 353-359

[26] Baldermann JC, Schuller T, Huys D et al. Deep Brain Stimulation for Tourette-Syndrome: A Systematic Review and MetaAnalysis. Brain stimulation 2016; 9: 296-304

[27] Verdellen C, Van De Griendt J, Hartmann A et al. European clinical guidelines for Tourette syndrome and other tic disorders. Part III: behavioural and psychosocial interventions. European child \& adolescent psychiatry 2011; 20: 197-207

[28] Dutta N, Cavanna AE. The effectiveness of habit reversal therapy in the treatment of Tourette syndrome and other chronic tic disorders: a systematic review. Functional neurology 2013; 28: 7

[29] Jakubovski E, Reichert C, Karch A et al. The ONLINE-TICS study protocol: a randomized observer-blind clinical trial to demonstrate the efficacy and safety of internet-delivered behavioral treatment for adults with chronic tic disorders. Frontiers in psychiatry 2016; 7: 119

Bibliografie

DOI https://doi.org/10.1055/a-0996-0944

Fortschr Neurol Psychiatr 2019; 87: 577-589

(c) Georg Thieme Verlag KG Stuttgart · New York ISSN 0720-4299 


\section{Punkte sammeln auf CME.thieme.de}

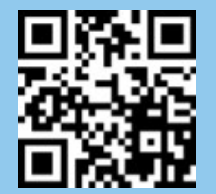

Diese Fortbildungseinheit ist in der Regel 12 Monate online für die Teilnahme verfügbar.

Den genauen Einsendeschluss finden Sie unter https://eref.thieme.de/CXDQGS2.

Sollten Sie Fragen zur Online-Teilnahme haben, finden Sie unter https://cme.thieme.de/hilfe eine ausführliche Anleitung. Wir wünschen viel Erfolg beim Beantworten der Fragen!

Unter https://eref.thieme.de/CXDQGS2 oder über den QR-Code kommen Sie direkt zur Startseite des Wissenstests.

VNR 2760512019156642066

\section{Frage 1}

Welche Aussage zum Tourette-Syndrom ist falsch?

A) Tics beginnen typischerweise im Kindesalter.

B) In der Anamnese sind sowohl motorische als auch vokale Tics zu eruieren.

C) Tics bestehen für einen Zeitraum von mindestens einem Jahr.

D) Psychiatrische Komorbiditäten sind häufig.

E) Koprolalie ist Teil der diagnostischen Kriterien.

\section{Frage 2}

Welche Aussage zu Tics ist richtig?

A) Eine Unterscheidung von Tics ist hinsichtlich Komplexität und Qualität möglich.

B) Naserümpfen ist ein komplex motorischer Tic.

C) Tics sind nicht unterdrückbar.

D) Tics verschlechtern sich unter Konzentration.

E) Echolalie beschreibt das Wiederholen selbst ausgesprochener Wörter / Satzfragmente.

\section{Frage 3}

Welche Aussage ist falsch? Zu häufigen psychiatrischen Komorbiditäten bei Tic-Störungen zählen. . .
A) $\ldots$ ADHS
B) ....Angststörung
C) ...Zwänge
D) ....artifizielle Störung
E) ...Depression

\section{Frage 4}

Welche Aussage bezüglich der Ätiologie des TouretteSyndroms ist falsch?

A) Es besteht eine Dysfunktion der kortiko-striato-pallidothalamo-kortikalen Regelkreise.

B) Die Ätiologie wird multifaktoriell erklärt.

C) Genetische als auch umweltbedingte Faktoren spielen eine wichtige Rolle.

D) Das dopaminerge System ist mitbetroffen.

E) Das Erkrankungsrisiko liegt bei $50 \%$, wenn ein Elternteil betroffen ist.

\section{Frage 5}

Welche Aussage zum Verlauf des Tourette-Syndroms ist richtig?

A) Vokale Tics treten in der Regel 2-3 Jahre vor motorischen Tics auf.

B) Das Ersterkrankungsalter beträgt 10 bis 11 Jahre.

C) Tic-Störungen haben einen abrupten Beginn, wobei die Tics kaum hinsichtlich ihrer Art und Häufigkeit variieren.

D) Tics treten typischerweise im Alter von 6 bis 7 Jahren zum ersten Mal auf.

E) Nur in äußerst wenigen Fällen kommt es zu einer Spontanreduktion der Tics im Erwachsenenalter.

\section{Frage 6}

Welche Aussage zur Therapie von Tic-Störungen ist richtig?

A) Tic-Störungen sollten immer nur psychopharmakologisch behandelt werden.

B) Tic-Störungen sollten immer nur psychotherapeutisch behandelt werden.

C) In vielen Fällen führt eine ausführliche Psychoedukation bereits zu einer deutlichen Entlastung der Patienten.

D) Antipsychotika sind Methode der ersten Wahl der Behandlung von Tic-Störung.

E) Neben Haloperidol sind in Deutschland ebenso die Medikamente Tiaprid, Aripiprazol und Risperidon zugelassen.

\section{Frage 7}

Welche Aussage zur Exposure and Response Prevention (ERP) ist richtig?

A) Hauptelement ist das Identifizieren und Einüben von Gegenbewegungen.

B) ERP eignet sich vor allem, wenn eine Tic-Störung durch viele verschiedene Tics gekennzeichnet ist und der Betroffene starke Vorgefühle verspürt.

C) Die ERP wird vor allem zur Behandlung von automatisierten und situationsspezifischen Tics herangezogen.

D) Die Behandlung mittels ERP beinhaltet mindestens 20 Sitzungen.

E) Hauptziel ist nicht die Reduktion der Tics, sondern die Erhöhung der Selbstwirksamkeit des Betroffenen. 


\section{Punkte sammeln auf CME.thieme.de}

Fortsetzung $\ldots$

\section{Frage 8}

Welche Aussage zum HRT / CBIT ist richtig?

A) Das HRT beinhaltet keine Psychoedukation.

B) Das HRT eignet sich insbesondere die Abfolge von Vorgefühl und Tic zu unterbrechen.

C) HRT und CBIT können auch angewendet werden, wenn in der Therapiestunde keine Tics vorliegen oder das Vorgefühl nur sehr schwach ausgeprägt ist.

D) HRT und CBIT können die Tics um $10-20 \%$ reduzieren.

E) Das HRT ist eine Erweiterung der CBIT um eine ausführliche Psychoedukation, ein Achtsamkeitstraining und Funktionsanalysen.

\section{Frage 9}

Auf welche der folgenden Neurotransmittersysteme zielen die bei Tic-Störungen eingesetzten Psychopharmaka eher nicht ab?
A) cholinerges System
B) dopaminerges System
C) serotonerges System
D) Cannabinoid-System
E) noradrenerges System

\section{Frage 10}

Welche Aussage zur Behandlung bei komorbiden Störungen und Therapieresistenz ist falsch?

A) Bei Therapieresistenz kann eine Tiefenhirnstimulation am Globus pallidus internus zu einer Tic-Reduktion führen.

B) Die noradrenerg wirksamen Substanzen Clonidin und Guanfacin können sowohl Tics als auch Symptome eines ADHS positiv beeinflussen.

C) Neben Antidepressiva sind Aripiprazol und Risperidon Alternativen bei komorbider Zwangssymptomatik.

D) Bei einer komorbiden ADHS ist Methylphenidat wegen des Risikos einer Tic-Verstärkung kontraindiziert.

E) Cannabinoide sind eine mögliche Alternative bei Versagen herkömmlicher psychopharmakologischer Therapieansätze. 